K.A. van der Hucht, G. Koenigsberger \& P.R.J. Eenens, eds.

\title{
Evolutionary population synthesis: the effect of binary systems
}

\author{
J. Miguel Mas-Hesse and Miguel Cerviño
}

\author{
LAEFF-INTA, P.O. Box 50727, E-28080 Madrid, Spain
}

\begin{abstract}
We present in this contribution our set of multi-wavelength synthesis models including the evolution of single and binary stars. The main results we have obtained can be summarized as follows: (a) massive close-binary systems will start to experience mass transfer episodes after the first $4 \mathrm{Myr}$ of the starburst evolution; (b) as a result of these mass transfer processes, stars of relatively low initial mass can lose completely their envelope and become a Wolf-Rayet star. In this way, the formation of WR stars is extended over longer than $15 \mathrm{Myr}$, and does not stop at $6 \mathrm{Myr}$ as predicted by models including only single stars; (c) WR stars can thus be coeval with red supergiants, which peak at around $10 \mathrm{Myr}$ for solar metallicities; (d) the accretion of mass will originate relatively massive stars at ages for which they should have already disappeared; these stars, together with the WR stars formed in rather evolved clusters, increase the production of ionizing photons, so that the $\mathrm{H} \beta$ equivalent width will not drop as rapidly as predicted by models considering only individual stars; and (e) the mass transfer to compact companions will produce an additional source of high-energy radiation in the form of high-mass X-ray binaries, not predicted either by standard synthesis models.
\end{abstract}

\section{Introduction}

In the last years convincing observational evidence has been collected about the presence of starburst regions in or around active Seyfert 2 nuclei (Heckman et al. 1997; González-Delgado et al. 1998, and references therein). It has been found that most of the UV light from these objects originates in (circum-)nuclear star formation sites; the possible connection between the nuclear activity and the properties of these starbursts is nevertheless still a matter of debate. According to the unified schemes of Seyfert galaxies, the active nucleus should be hidden by an opaque torus in the case of Seyferts 2 , thus explaining why the UV light collected is indeed dominated by these young, massive stars. The low degree of contamination by the active source in the UV range allows, therefore, to get detailed information about the properties of the star formation processes. Moreover, extrapolating to the radio-to-X-ray ranges the emission associated with the starbursts, it should be possible to disentangle the fractional contribution of both sources (the starburst and the active nucleus) at different energy ranges. Multi-wavelength evolutionary synthesis models normalized to the observed UV emission would be the ideal tool to perform this analysis.

With these ideas in mind, we started some years ago a program to extend our evolutionary synthesis models to high energy ranges (soft and hard X-rays, gamma-rays). Preliminary results were presented in Cerviño et al. (1996). It 
was clear that to properly reproduce the high energy emission, the effects of mechanical energy release (by stellar winds and supernova explosions) on heating the diffuse interstellar gas had to be included, as well as the evolution of binary systems. The process of mass transfer between close interacting companions can affect the evolution of the individual stars, and can also trigger high energy emission when one of the stars has already evolved to a compact object. Binaries have also been included in starburst computations by Vanbeveren et al. (1997) and Van Bever \& Vanbeveren (1998). Schaerer \& Vacca (1998) performed also a simple approximation to the effects of binary systems on the evolution of young starbursts. In recent reviews by Vanbeveren et al. (1998, these Proceedings), a detailed description of the evolution of massive close binary systems has been presented.

We present in this contribution our set of stellar population models including the evolution of single and binary stars. We will stress the effects of binary evolution on the stellar population structure. In Sect. 2 we present our set of evolutionary synthesis models and in Sect. 3 we discuss the predictions on the stellar population. In an accompanying contribution in these Proceedings (Cerviño \& Mas-Hesse: Hard X-ray-to-radio energy distributions in starburst galaxies), the multi-wavelength spectral energy distribution and the effects on the $\mathrm{H} \beta$ equivalent width and HeII emission line are discussed. The complete set of models will be published elsewhere.

\section{Evolutionary synthesis models for single and binary stars}

The basic ideas about our evolutionary synthesis models for single stars have already been discussed in Mas-Hesse \& Kunth (1991, hereafter MHK) and in Cerviño \& Mas-Hesse (1994, hereafter CMH). Basically, our models are based on different Initial Mass Function (IMF) slopes ( $\alpha=1,2.35$ (Salpeter) and 3), two extreme star formation regimes (Instantaneous (IB) and Extended Bursts (EB) and five sets of stellar evolutionary tracks with different metallicities taken from the Geneva group (Schaller et al. 1992 and references therein). In this section we discuss the framework we have used to include the evolution of binary systems, which is based on the prescriptions of Vanbeveren (1991) and Vanbeveren et al. (1998), somewhat modified and simplified.

We assume that the stars evolve like single, non-rotating stars following their theoretical tracks until mass transfer episodes or supernova explosions occur. The procedure for track interpolation in mass has been discussed in detail in $\mathrm{CMH}$. We want to remark that we compute the evolution of each individual mass along the HR diagram, and obtain at each time step the total number of stars and their luminosity for each spectral type and luminosity class bin. In case of the stars forming a binary system, we re-compute continuously their mass ratio and orbital separation using their instantaneous masses, as defined by the stellar tracks. We have assumed circular orbits along the evolution of the systems, although we have also considered the possible formation of very eccentric systems after a supernova explosion. 


\subsection{Initial distributions}

The parameters determining the evolution of binary systems are mainly the mass of each component, their mass ratio $q\left(=M_{2} / M_{1}\right.$ where $M_{1}$ corresponds to the more massive star) and the orbital semi-axis, $a$ or period $P$, together with the fraction of stars formed in such systems. The initial distributions of these parameters will define the evolution of the young clusters and their observational properties.

We have assumed by default that around $50 \%$ of the stars are formed in binary systems, supported by the frequency of WR stars in binaries obtained by Vanbeveren \& Conti (1980) for the solar neighbourhood. Nevertheless, we have also explored the effect of assuming different binary frequencies $(10,30,70$ and $90 \%$ ). In general, even assuming low frequencies around $10 \%$, the effects on the evolution of the stellar population are already remarkable. We want to stress that this binary frequency $f$ refers to the total number of binary systems, and not just to those experiencing mass transfer. Binary interactions will affect indeed only less than $5 \%$ of all systems (Maeder \& Meynet 1994).

The IMF is generated by a Montecarlo technique, as explained in Arnault (1990), which provides an initial mass value for each star formed in the cluster. It is forced to follow a power-law with three fixed values of the slope. The mass ratio distribution has been obtained from the same Montecarlo run, by randomly associating two subsequent mass values, only constrained to comply with the defined binary frequency. Finally, the orbital separation distribution has been also generated by a Montecarlo routine, forced to a $d N / d a \propto a^{-1}$ function. In order to have statistical significance, we have used a distribution of $5 \times 10^{5}$ stars (i.e., $1.25 \times 10^{5}$ binary systems). As explained in $\mathrm{CMH}$, smaller distributions of stars can lead to significant stochastic variations in the stellar population from one Montecarlo run to another. While this feature reproduces what we observe in small clusters, for the purpose of this work we have assumed only large numbers of stars with well defined initial input distributions. The initial distributions we have assumed are summarized in Table 1.

Table 1. Initial input parameter distributions used in the models.

\begin{tabular}{lll}
\hline input parameter & law & method \\
\hline IMF & $\begin{array}{l}d N / d m \propto m^{-\alpha}(\alpha=1,2.35,3) \\
\text { mass limits: } 2-120 \mathrm{M}_{\odot}\end{array}$ & Montecarlo \\
star formation regime & instantaneous, extended & \\
binary frequency & $50 \%(10,30,70,90 \%)$ & Montecarlo \\
mass ratio & $q=M_{2} / M_{1}$ & Montecarlo \\
orbital separation & $d N / d a \propto a^{-1}$ & \\
& $\frac{a_{\min }}{\mathrm{R}_{\odot}}<6\left(\frac{M_{1}}{\mathrm{M}_{\odot}}\right)^{1 / 2}, \frac{a_{\max }}{a_{\min }}=5000$ & \\
\hline
\end{tabular}




\subsection{Roche Lobe over-flow phase (RLOF)}

Mass transfer episodes during Roche lobe over-flow are the most critical process when analyzing the evolution of binary systems. We test continuously whether RLOF episodes occur by comparing the Roche lobe size with the stellar radii at each time step. Vanbeveren et al. (1997) show that systems in which the primary has an initial mass larger than $40 \mathrm{M}_{\odot}$ with periods such that RLOF should start during or after the LBV phase, can avoid the mass transfer episode due to the loss of mass via stellar winds, so that no interaction will occur. We have therefore neglected possible mass transfer episodes in stars initially more massive than $40 \mathrm{M}_{\odot}$ or when the primary has reached the WR phase when RLOF should occur. This restriction has important consequences, in the sense that the properties of a cluster containing binary systems will not differ significantly from a singlestars-only cluster during the first Myr of evolution, until the most massive stars in the clusters explode as supernovae and might originate binary systems with a compact companion. When RLOF occurs, the evolution of the donor and gainer remnants will depend mostly on their initial mass, their evolutionary status, and the amount of mass transferred:

- Donor star. The donor star will generally lose its outer layers during mass transfer episodes. Depending on its structure and evolutionary status we can identify three main cases:

i. The Donor is an H burning star (Case A). In this situation mass transfer will last until $\mathrm{H}$ is exhausted in the nucleus of the donor star, so that the transfer episode will be extended over the remaining main sequence lifetime of the star. As a result, the donor lifetime will be longer than that of a single star with the same initial mass. As a net effect of Case A episodes the mass ratio of the system will be reversed, and the individual stars will continue their evolution according to their new mass, considering that around $50 \%$ of the mass has been assumed to be lost during the transfer episode. We show in Fig. 1a the evolution of a system experiencing Case A mass transfer.

ii. Donor between $H$ and He burning phase (Case B). In this case, the star looses mass until the atmospheric $\mathrm{H}$ abundance is around 0.2 (de Greve $\&$ de Loore 1992). The remnant mass will be, according to Vanbeveren et al. (1998):

$$
m_{d}^{\text {post RLOF }}=0.093 \times\left(M_{d}^{\text {pre RLOF }}\right)^{1.44}
$$

As a first approximation we have assumed this law for all metallicities considered, but it may change with metallicity as discussed by Vanbeveren et al. (1998). If the remnant mass of the donor star is larger than $5 \mathrm{M}_{\odot}$, it becomes a WR-like star, according to Vanbeveren et al. (1997). Bare He burning stars formed after RLOF and with less than $5 \mathrm{M}_{\odot}$ have been counted also as Wolf-Rayet stars, although their expected weaker, but non-zero, stellar winds might not be able to form a thick mantle originating the spectral features associated with WRs. The value of this mass limit will be refined in the future, when more precise atmosphere models for this kind of stars become available. In the meantime we want to stress 

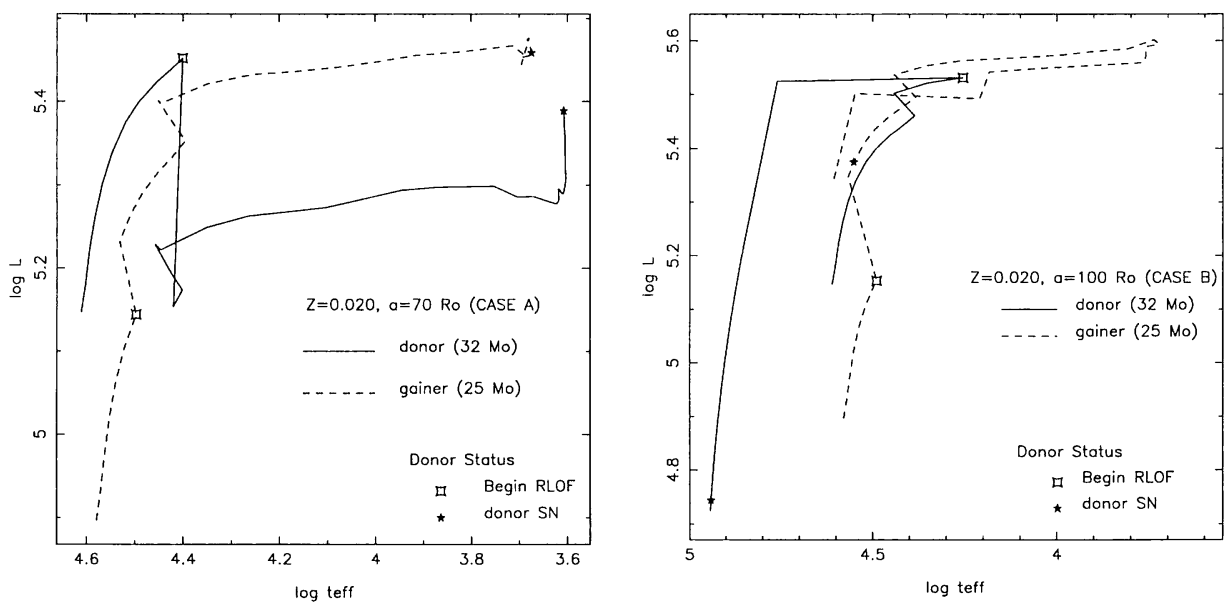

Figure 1. Evolution of a binary system (a) following Case A and (b) following Case B of mass transfer.

that the WR population our models are predicting at evolved ages (longer than $11 \mathrm{Myr}$ ) does not necessarily show the same spectral features than more massive WRs.

In order to follow the evolution of the star after mass transfer, we have assumed that: the evolutionary status remains the same (no time correction); the surface abundances are equal to those in the core; the instantaneous mass is re-calculated at each time step taking into account the remnant mass and the mass loss laws for the different WR phases; the WNL phase will last until all the $\mathrm{H}$ is removed from the envelope, assuming a mass loss rate of $4 \times 10^{-5} \mathrm{M}_{\odot} \mathrm{yr}^{-1}$; and the WC phase will follow the massluminosity and mass-radius relations from Schaerer \& Maeder (1992) for WR stars and Langer (1989) for He stars (mass after RLOF lower than $5 \mathrm{M}_{\odot}$ ). In Fig. $1 \mathrm{~b}$ we show the evolution of a system experiencing a Case $\mathrm{B}$ RLOF episode.

iii. Donor in He-burning or later phases (Case C). In this case the effects of shell-burning regions in the structure of the star are not clear, so that the evolution after RLOF is quite uncertain. Anyway, since the donor remnant will be already very evolved, its remaining lifetime will be very short and these systems will be a minority (Vanbeveren et al. 1997). We have therefore not considered this case of mass transfer episodes in our models.

- Gainer star. We have assumed that the gainer star will be able to accrete the mass lost by the donor if it is still in the Main Sequence, with a convective envelope. In such cases we have considered that $50 \%$ of the mass and angular momentum of the system is lost anyway by the system in the mass transfer process (Meurs \& van den Heuvel 1989). The gainer will become a more massive 

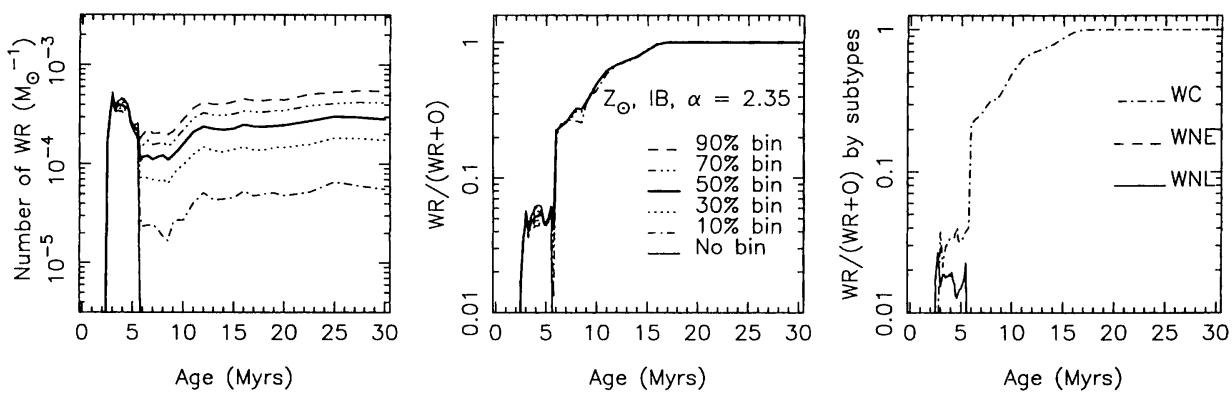

Figure 2. (a) Predicted number of WR stars, normalized by the mass of stars formed in the cluster; (b) WR over WR+O stars. Note that the ratio is almost independent on the binary abundance; and (c) WR types.

star, which will evolve as a younger one. Combining the evolutionary status of the star before the RLOF episode and the amount of mass accreted, we compute a time correction and make the star evolve following the track of a younger, more massive star. The net effect of these processes will be the 'rejuvenation' of the cluster (Van Bever \& Vanbeveren 1998), which will host massive stars at ages where all of them should have already exploded as SN, if only single stars where present.

If the gainer is in more evolved phases when RLOF occurs, we have assumed that it will not be able to accrete mass, and that all the mass transferred is lost by the system. In these cases common envelope evolution and spiral-in processes might become important.

\subsection{SN explosions and formation of high mass X-ray binaries}

After a supernova explosion, the binary system will survive only if the mass of the non-exploding star is two times larger than the mass of the exploding one. We test this condition when each of the massive stars in the synthetic cluster exhausts its nuclear fuel, and compute then the number of systems that will remain bound or become unbound after the SN explosion. If the exploding star had at the moment of the explosion a mass higher than $8 \mathrm{M}_{\odot}$, we assumed that it becomes a black hole with more than $4 \mathrm{M}_{\odot}$. If the mass is lower than 8 , but higher than $4 \mathrm{M}_{\odot}$, it will become a neutron star with around $3 \mathrm{M}_{\odot}$. Otherwise, it will become a white dwarf with $1.4 \mathrm{M}_{\odot}$. After the SN explosion the system might become very eccentric, depending on its mass ratio and orbital separation before the explosion. We have assumed that these eccentric post-SN systems might become a $\mathrm{Be} / \mathrm{X}$ star (highly eccentric systems with a main sequence star plus a compact companion). As the remnant star evolves, it will become a supergiant. Then we assume that the orbit will be circularized, giving rise to a 'permanent' high mass X-ray binary (HMXRB). 

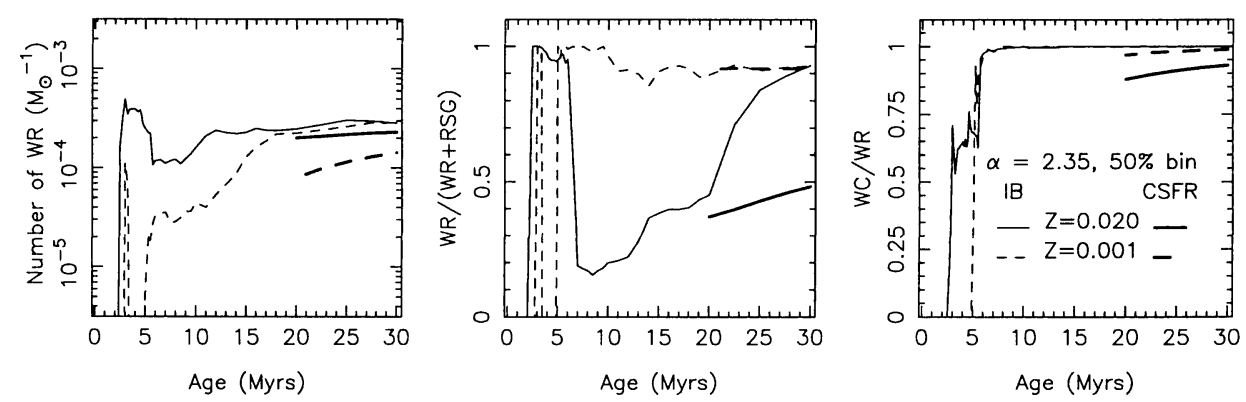

Figure 3. (a) Predicted WR population for two metallicities. (b) WR over WR + RSG ratio. (c) WC over WR ratio. Predictions for continuous star formation rate (CSFR) have also been included.

\section{Model predictions}

We will summarize shortly in this section the most relevant predictions of our evolutionary models affected by the presence of binary systems.

- Wolf-Rayet stars population. One of the most relevant effects of the mass transfer processes in close binaries is the generation of WR stars at ages where models based only on single stars do not predict the presence of any WR at all. We show in Fig. 2 the predicted number of WRs, as well as their types and ratios over O-type stars, as a function of the binary frequency. It can be seen that while the generation of WR stars from the evolution of single stars remains essentially the same, after around $5 \mathrm{Myr}$ a relatively large number of WR stars is originated in close binary systems. We want to stress that massive stars in detached binary systems will evolve essentially as single stars, and that this new population of WR stars is related only to the binary systems experiencing interactions.

In Fig. 3 we show the dependence of the WR population properties on metallicity. It is interesting to see that the formation of WRs by the binary channel is also metallicity dependent, as a consequence of the different evolution of single stars at lower metallicities. An important result of our models is the prediction of the simultaneous presence of WR and red supergiant (RSG) stars in evolved (older than $7 \mathrm{Myr}$ ) clusters formed at metallicities above $Z \simeq 0.008$.

Finally, it is also important to see that our models predict that the WR stars formed by the binary channel will be preferentially of the WC type. As explained above, WR stars formed in Case B will lose their envelope and will show a surface abundance similar to that in the nucleus, i.e., rich in carbon.

- $O B$ stars and $W(\mathrm{H} \beta)$. The accretion of mass by transfer episodes will originate continuously a population of relatively massive stars at ages where they should have already disappeared from the young clusters (assuming nearly instantaneous bursts). These stars, together with the hot WR stars formed, will maintain the ionizing power of the clusters at a significantly higher level than just single stars. As a result, the strength of the emission lines will decrease 
with time much slower. With a binary frequency of $50 \%, W(\mathrm{H} \beta)$, frequently used as an age estimator, will be larger by more than an order of magnitude at 15 Myr than the value predicted for single stars clusters.

- Systems with compact objects. The fast evolution of the massive primary originates the formation of close systems with a compact (black hole, neutron star) component after the first 4-5 Myr of evolution. The accretion of mass onto the surface of the compact companion will give rise to high-energy phenomena. As an example, we predict around $10 \mathrm{HMXRB}$ to be present in a $15 \mathrm{Myr}$ cluster having formed around $10^{7} \mathrm{M}_{\odot}$ of stars.

- Spectral energy distribution (SED). The SED will be drastically affected by the presence of binary systems in the cluster. The formation of HMXRB and related objects provides a source of high-energy emission which completely dominates the hard X-ray range (above around $2 \mathrm{keV}$ ) after the first 4-5 Myr. More details are given in the accompanying contribution by Cerviño \& MasHesse (these Proceedings).

Acknowledgments. This work has been supported by Spanish CICYT ESP-95-0389-C02-02 and INTA 'Rafael Calvo Rodés' grants.

\section{References}

Arnault, Ph. 1990, Ph.D. Thesis, Institut d'Astrophysique de Paris

Cerviño, M., Mas-Hesse, J.M. 1994, A\&A 284, 749 (CMH)

Cerviño, M., Mas-Hesse, J.M., Kunth, D. 1996, in: J.-M. Vreux, A. Detal, D. FraipontCaro, E. Gosset \& G. Rauw (eds.), Wolf-Rayet Stars in the Framework of Stellar Evolution, Proc. 33-rd Liège Int. Astroph. Coll., (Liège: Univ. of Liège), p. 613,

De Greve, J.P., de Loore, C.W.H. 1992, A\&AS 96, 653

González-Delgado, R., Heckman, T., Leitherer, C., Meurer, G., Krolik, J., Wilson, A.S., Kinney, A., Koratkar, A. 1998, ApJ 505, 174

Heckman, T.M., González-Delgado, R., Leitherer, C., Meurer, G., Krolik, J., Kinney, A., Koratkar, A., Wilson, A.S. 1997, ApJ 482, 114

Langer, N. 1989, A\&A 210, 93

Maeder, A., Meynet, G. 1994, A\&A 287, 803

Mas-Hesse, J.M., Kunth, D. 1991, A\&AS 88, 399 (MHK)

Meurs, E.J.A., van den Heuvel, E.P.J. 1989, A\&A, 226, 88

Schaerer, D., Maeder, A. 1992, A\&A 263, 129

Schaerer, D., Vacca, W.D. 1998, ApJ 497, 618

Schaller, G., Schaerer, D., Meynet, G., Maeder, A. 1992, A\&AS 96, 269

Van Bever, J., Vanbeveren, D. 1998, A\&A 334, 21

Vanbeveren, D., Conti, P.S. 1980, A\&A 80, 230

Vanbeveren, D. 1991, A\&A 252, 159

Vanbeveren, D., Van Bever, J., de Donder, E. 1997, A\&A 317, 487

Vanbeveren, D., de Donder, E., Van Bever, J., Van Rensbergen, W., de Loore, C.W.H. 1998, New Astronomy 3, 443 


\section{Discussion}

Vanbeveren: The number of estimated HMXBs depends critically on the adopted asymmetry of the SN explosions of a massive star, which effects critically the disruption probability of a binary during the SN explosion in one of the components. How do you treat these effects in your population synthesis models?

Mas-Hesse: This will be explained in detail in the written contribution to the Proceedings.

Walborn: Your HST-WFPC2 image of NGC 4214 is fantastic! I can't emphasize its importance as an intermediate stepping stone between the nearby starbursts such as 30 Dor and N 11, and more distant, larger, unresolved regions. In NGC 4214 two "N11's" and several "30 Dor's" are resolved, showing that the global starburst consists of discrete regions with two-stage structures of different ages.

Mas-Hesse: I agree with you. About the NGC 4214 HST image, it was color-coded by J. Maíz-Apellániz by combining continuum and emission-line HST archival images.

Maeder: In the comparisons of starburst galaxies and population synthesis, we often mix a lot of different problems: stellar models, binary frequencies, number if ionizing photons, dilution factors, geometrical effects, etc. For me, I consider we can apply population syntheses to distant galaxies and starbursts, only when they have been very carefully checked for consistency with open star clusters in the Galaxy, the LMC and the SMC.

Mas-Hesse: In my opinion we should follow both approaches in parallel, with mutual feedbacks in both directions.

Leitherer: The SSCs in NGC 4214 and NGC 1705 are ideal targets to test single/binary models. They are single-stellar populations $(D \simeq$ a few pc) and were studied spectroscopically with HST and Keck. The NGC 4214 cluster is 4-5 Myr old (from UV-line spectroscopy), has WR stars but no RSGs (the latter from Keck). The NGC 1705 cluster was observed with the same instruments. It has few if any $\mathrm{O}$ and WR stars, but a significant RSG population. Can these results be used as constraints?

Mas-Hesse: This is indeed the way to follow in the future, but better statistics than just one case is clearly needed. There are many emission-line galaxies with estimated burst ages around 4-6 Myr which don't show evidences of WR stars either, but no one would invalidate the Conti-scenario because of this.

Langer: One important effect of binaries in your models is the appearance of WR stars for up to $30 \mathrm{Myr}$. I think these 'late' WR stars must all be of low mass, i.e., with actual masses below $5 \mathrm{M}_{\odot}$ or so. Now, as far as I know, such WR stars are not observed in the Galaxy nor the Magellanic Clouds (nor elsewhere). What do you assume for their spectral energy distribution, or how do you think can they be observable in the integral light of a starburst at all?

Mas-Hesse: The WR population that we predict after around $10 \mathrm{Myr}$ contains indeed many stars with masses below $5 \mathrm{M}_{\odot}$. They are naked He-stars of high temperature, but low luminosity. As a first approximation, we have included their contribution considering their luminosity and effective temperature, as predicted by the evolutionary tracks, assuming their line features should be similar to those of average WC stars. It is clear that we need specific atmosphere models for these stars in order to be able to predict their real observational properties. 